\title{
Analysis of Zeno Behaviors in Hybrid Systems*
}

\author{
Michael Heymann† Feng Lin; George Meyer ${ }^{\S}$ and Stefan Resmerita
}

\begin{abstract}
In this paper, we investigate conditions for existence of Zeno behaviors in hybrid systems. These are behaviors that occur in a hybrid system when the system undergoes an unbounded number of discrete transitions in a finite and bounded length of time. Zeno behavior occurs, for example, when a controller unsuccessfully attempts to satisfy an invariance specification by switching the system among different configurations faster and faster. Two types of Zeno systems will be investigated: (1) strongly Zeno systems where all runs of the system are Zeno; and (2) (weakly) Zeno systems where only some runs of the system are Zeno. We derive necessary and sufficient conditions for both strong Zenoness and Zenoness, under certain assumptions. Our analysis is based on studying the trajectory set of a certain "equivalent" continuous-time system that is associated with the dynamic equations of the hybrid system. We also study the relation between the possibility of existence of Zeno behaviors in a system and the problem of existence of non-Zeno safety controllers that prevent the system from entering a suitably defines illegal region of its operating space. In particular, we show that if the system is Zeno (but not strongly Zeno), then a minimally-interventive safety controller may not exist, even if a safety controller does exist, disproving a conjecture made earlier in the literature. We also argue that any attempt of "regularizing" Zeno systems by forcing delays between successive configuration switches will not be fruitful.
\end{abstract}

Keywords: Hybrid systems, Zenoness, control

${ }^{*}$ This research is supported in part by NSF under grant ITR-0082784 and NASA under grant NAG2-1043 and in part by the Technion Fund for Promotion of Research. The work by the first author was completed while he was visiting NASA Ames Research Center, Moffett Field, CA 94035, under a grant with San Jose State University.

${ }^{\dagger}$ Department of Computer Science, Technion, Israel Institute of Technology, Haifa 32000, Israel, e-mail: heymann@cs.technion.ac.il

${ }^{\ddagger}$ Department of Electrical and Computer Engineering, Wayne State University, Detroit, MI 48202, USA, and School of Electronics and Information Engineering, Tongji University, Shanghai, China, e-mail: flin@ece.eng.wayne.edu

§NASA Ames Research Center, Moffett Field, CA 94035, e-mail: George.Meyer-1@nasa.gov

"Department of Computer Science, Technion, Israel Institute of Technology, Haifa 32000, Israel, e-mail: stefan@cs.technion.ac.il 


\section{Introduction}

Zenoness $^{1}$ is a phenomenon unique to hybrid systems. It describes the situation where a hybrid system undergoes an unbounded number of discrete transitions in a finite and bounded length of time. This can happen, for example, when a (discrete) controller unsuccessfully attempts to satisfy an invariance specification by switching the system faster and faster among distinct configuration. Zenoness can be thought of as a type of instability of hybrid systems that constitutes a major impediment to "proper" system behavior.

In recent years, various algorithms have been proposed in the literature for synthesis of controllers for hybrid systems aimed at achieving specified state-space invariance condtions $[3,4,5,6,9,10,12,11,13,15,19,25]$. All these algorithms are based on the implicit assumption that if the invariance condition can be satisfied by some controller, the synthesis algorithm will converge successfully to a maximal invariant set, and hence yield an optimal (minimally interventive) controller. While this is true when the underlying system is nonZeno (i.e., when non of its behaviors is Zeno), as was shown explicitly in [11], the question as to whether a (minimally interventive) safety controller exists when the system is Zeno was not resolved.

Since Zenoness is quite a complex phenomenon, several researchers have proposed controller synthesis approaches aimed at bypassing the difficulties created by the possible presence of Zeno behaviors. Specifically, the maximal switching rate of the synthesized controller was limited by imposing various structural constraints on either the system or on the controller; thereby yielding controlled systems that switch configurations at or below a specified finite upper rate $[4,5,9,25]$. Yet, while such approaches guarantee that a synthesized controller will never yield a Zeno system, they do not answer the basic questions associated with the Zenoness phenomenon. In particular, when controllers with the imposed switching rate constraint exist, are they necessarily minimally interventive for the system when no switching rate constraints are imposed? When controllers with the imposed switching rate constraint do not exist, what conclusions can be drawn regarding the existence and nature of controllers for the unconstrained system? Are Zeno behaviors inherently possible in the unconstrained system? When a safety controller for the constrained system exists, does there also exist a minimally interventive controller for the unconstrained one? If the answer to this latter question is affirmative, how are the two controllers related? These questions cannot be answered by forcing delays between successive configuration switches and viewing the Zenoness issue as an annoyance.

To address the above questions, it is first of all, necessary to establish conditions under which a hybrid system exhibits Zeno behaviors and, hence, to derive necessary and sufficient conditions for their occurrence. This is the main focus of the present paper. Conditions for Zenoness were investigated in [26], [27] and [24] for certain classes of hybrid systems. These

\footnotetext{
${ }^{1}$ After the Greek philosopher Zeno whose famous paradox about the race between Achillis and the tortoise resembles the said behavior.
} 
are hybrid systems that usually have state resets, and it was shown that the nature of the Zeno behaviors may depend strongly on properties of the state reset-map. In the present paper we examine a special class of a hybrid system that we call homogeneous. These are hybrid systems where the (continuous) state variables represent similar (and frequently the same) physical objects. Thus the state variables are of the same dimension in all (discrete) configurations and they are never reset. Systems of this type have been investigated in [20], [22] and [23], with particular emphasis on stability, periodicity and existence of limit cycles of solutions. In this paper we distinguish between two types of Zenoness: strong Zenoness when all of the system's runs are Zeno, and (weak) Zenoness when only some of its runs are Zeno. We derive necessary and sufficient conditions for Zenoness and strong Zenoness for a class of homogeneous systems. For homogeneous hybrid systems with constant-rate dynamics, we find a necessary and sufficient condition for strong Zenoness. Furthermore, if a system is also regular, that is, if the number of state variables is equal to the number of discrete configurations, we find a necessary and sufficient condition for Zenoness. Both conditions can be easily checked based on the matrix describing the rates of change of the state variables in all configurations. The results are extended to bounded-rate hybrid systems as well. These are hybrid systems where only upper and lower bounds of the rates are known. The Zenoness conditions for constant and bounded-rate hybrid systems can be employed for the study of a class of nonlinear hybrid systems (that can be approximated by constant-rate systems in the vicinity of the origin). We briefly discuss this approximation.

Our approach for finding necessary and sufficient conditions for Zenoness is based on a simple but crucial observation that a state of the hybrid system is reachable at a given time if and only if it is reachable at the same time in an "equivalent" continuous system that is obtained as a suitable weighted combination of the dynamic equations of the hybrid system in the different configurations. Thus, instead of a difficult investigation of the rather complicated class of behaviors of the hybrid system, we examine the very simple class of behaviors of the corresponding continuous system.

In this paper, we also investigate the relation between the possibility of existence of Zeno behaviors in a system and the problem of existence of viable (that is, non-Zeno) safety controllers for the system. Obviously, if the system is strongly Zeno, then no viable controller exists. More interestingly, we show that if the system is Zeno (but not strongly Zeno), then a minimally interventive safety controller may not exist even if a safety controller does exist.

\section{Notations and Motivation}

We consider hybrid systems $\mathcal{H}$ that consist of $n$ discrete configurations $\left\{q_{1}, q_{2}, \ldots q_{n}\right\}$, where at each configuration $q_{i}$ the continuous dynamics is given by $\dot{x}^{i}=f_{i}\left(x^{i}, u\right)$, where $x^{i}$ is an $m$ dimensional state vector $x^{i}=\left(x_{1}^{i}, \ldots, x_{m}^{i}\right)$. We assume that the state vectors $x^{i}$ represent similar (or the same) physical objects or phenomena in all configurations and hence are 
simply denoted by $x$. The initial value of $x$ is given by $x_{0}$ and the state vector is never reset (re-initialized). We call a system satisfying these conditions a homogeneous hybrid system.

As stated, we consider homogeneous hybrid systems with $n$ configurations and $m$ continuous variables. Each configuration is guarded by a set of predicates (guards) that specify the legal region in the state space $\mathbb{R}^{m}$ in which the state vector $x$ must remain at all time. When a guard (a predicate) becomes true, a transition is triggered to another (specified) configuration.

A transition $e \in E$ from a configuration $q$ to a configuration $q^{\prime}$ can be triggered either by a guard $G$ becoming true (and henceforth called a dynamic transition and denoted by $q \stackrel{G}{\longrightarrow} q^{\prime}$ ), or by a control (input) event $\underline{\sigma}$ (called an event transition and denoted by $q \stackrel{\sigma}{\longrightarrow} q^{\prime}$ ).

A run of a hybrid system $\mathcal{H}$ is a sequence

$$
q_{0} \stackrel{e_{1}, t_{1}}{\longrightarrow} q_{1} \stackrel{e_{2}, t_{2}}{\longrightarrow} q_{2} \stackrel{e_{3}, t_{3}}{\longrightarrow} \ldots
$$

where $e_{i}$ is the $i$ th transition and $t_{i}\left(\geq t_{i-1}\right)$ is the time when the $i$ th transition takes place. For each run, we define its time stamp, trajectory, and path as follows.

- The time stamp of the run is an n-dimensional (column) vector function $\operatorname{In}(t), t \geq 0$. If at time $t \geq 0 \mathcal{H}$ is in the $i$ th configuration, then $\operatorname{In}(t)$ has value 1 in its $i$ th entry and zeros in all others.

- For a run that starts at the initial state $x(0)=x_{0}$, the dynamics of $x(t)$ for $t \geq 0$ can then be expressed as

$$
\dot{x}=F(x, u, t):=\left[f_{1}(x, u) f_{2}(x, u) \ldots f_{n}(x, u)\right] \operatorname{In}(t),
$$

The solution to the above differential equation is the trajectory of the run.

- The path of the run is the sequence of the associated configurations.

We shall call a run of a hybrid system dynamic if all its transitions are dynamic transitions. An unbounded dynamic run

$$
q_{0} \stackrel{e_{1}, t_{1}}{\longrightarrow} q_{1} \stackrel{e_{2}, t_{2}}{\longrightarrow} q_{2} \stackrel{e_{3}, t_{3}}{\longrightarrow} \ldots
$$

is called a Zeno run if

$$
\lim _{i \rightarrow \infty} t_{i}=T<\infty
$$

A hybrid system is called Zeno if it possesses Zeno runs. Otherwise it is called non-Zeno or viable. A hybrid system all of whose runs are Zeno is called strongly Zeno.

To illustrate some aspects of the Zeno phenomenon, let us examine the following example. 


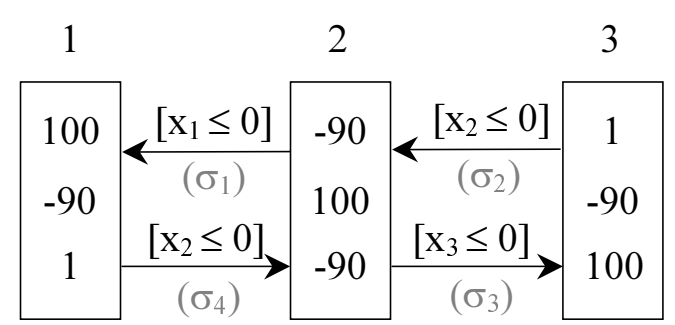

(a) The hybrid system

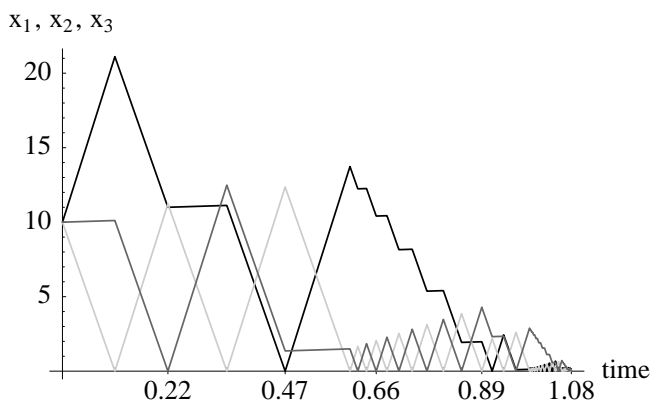

(c) The state variables

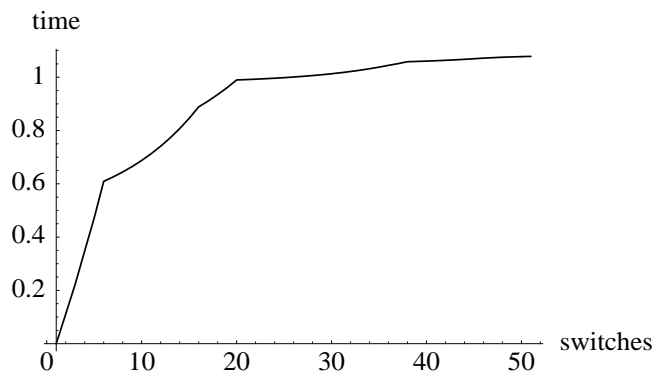

(b) Infinite switches occur in bounded time

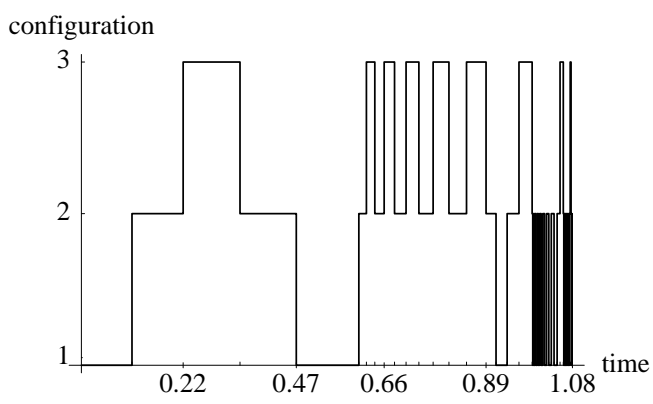

(d) The switching

Figure 1: Example of a Zeno system

Example 1 Consider the hybrid system shown in Figure 1(a). It consists of three configurations labeled by 1,2 , and 3 . There are three continuous variables $x_{1}, x_{2}$, and $x_{3}$. The rates of changes of these variables are displayed in each configuration (thus, in configuration $1, \dot{x_{1}}=100, \dot{x_{2}}=-90, \dot{x_{3}}=1$, etc.). When a variable reaches some lower bound ${ }^{2}$ and the corresponding guard becomes true, a dynamic transition is triggered that takes the system to a different configuration (e.g., when $x_{2}$ becomes zero in configuration 1, a transition is triggered to configuration 2) as shown in Figure 1(a).

Note that in each configuration of the system, at least one variable is decreasing and will eventually cause the system to change configuration. We call such a variable an active variable.

This example is an extension of the two water-tank example that we proposed in [12] and was also used by others [14]. However, the behavior of this system is much more complex than the two-water-tank example, as can be seen in Figure 1. It is not very straightforward to deduce intuitively from the dynamics whether the system is Zeno. Indeed, the switching among the three configurations is highly irregular as shown by the simulation results in Figure 1(d) and the "water level" in each tank (the value of the variables) does not show an obvious pattern as can be seen in Figure 1(c). However, as can be seen in Figure 1(b), an

\footnotetext{
${ }^{2}$ Without loss of generality, we assume that the lower bounds are 0 in this paper.
} 
unbounded number of transitions takes place in a finite length of time and hence the system is Zeno.

We are motivated, by this simple example and many others, to investigate the complex phenomenon of Zenoness. The first question that we would like to answer is how to check whether a system is Zeno or not, and the related question whether a safety controller exists.

\section{Conditions for Zenoness of Constant-Rate Systems}

To examine the Zenoness phenomenon and its relation to control synthesis, we review the concept of instantaneous configuration cluster (ICC) [12]. Let $v=\left[s_{1}, \ldots, s_{m}\right]$ be a valuation of the state vector. Let $\mathcal{H}(\sqsubseteq)$ denote the hybrid system obtained from $\mathcal{H}$ by deleting all dynamic transitions $q \stackrel{G}{\longrightarrow} q^{\prime}$ such that $G$ is not true for $v$. A strongly connected component $(\mathrm{SCC})^{3}$ of $\mathcal{H}(\sqsubseteq)$ that consists of two or more configurations is called an ICC. The triggering value $v$ of the state vector is called a Zeno point of $\mathcal{H}$.

In [12] it was shown that existence of a Zeno point and its associated ICC is a necessary condition for Zenoness, although it is not sufficient. Clearly, once at a Zeno point, the behavior of the hybrid system is necessarily Zeno. Thus, the question that must be examined is whether if initialized outside (or away from) a Zeno point, there exists a run that will converge to the Zeno point in a finite length of time, in which case we shall say that the Zeno point is a Zeno attractor. Clearly, a hybrid system is non-Zeno if and only if it has no Zeno attractor. Thus, the problem of checking Zenoness of a hybrid system consists of identifying its ICCs, if any, and checking whether they include Zeno attractors. In this paper, we address only the latter issue.

\subsection{General Constant-Rate Systems}

In this section we confine our attention to homogeneous constant-rate hybrid systems where, for real constants $k_{i j}$, the continuous dynamics in configuration $j, j=1,2, \ldots, n$, is given by

$$
\dot{x}=\left[\begin{array}{c}
\dot{x}_{1} \\
\dot{x}_{2} \\
\ldots \\
\dot{x}_{m}
\end{array}\right]=\left[\begin{array}{c}
k_{1 j} \\
k_{2 j} \\
\ldots \\
k_{m j}
\end{array}\right] .
$$

We shall further assume, that the systems under consideration satisfy the following assumption:

\footnotetext{
${ }^{3} \mathrm{An}$ SCC is a set of configurations for which there is a directed path from any configuration to any other.
} 


\section{Assumption 1}

(1) The legal region of the system is the nonnegative orthant $\mathbb{R}_{+}^{m}=\left\{x \in \mathbb{R}^{m}: x_{i} \geq 0, i=\right.$ $1,2, \ldots, m\}$.

(2) All the system's configurations are in an ICC with respect to the Zeno point $x=0$.

(3) Every variable is active in some configurations.

(4) In every configuration, there is at least one active variable.

(5) In a given configuration, a unique transition is associated with each active variable $x_{i}$. This transition is triggered either by an event (generated by a controller) or by the associated guard $\left[x_{i} \leq 0\right]$ becoming true. Each transition leads the system to a configuration where the triggering variable $x_{i}$ is not active.

In the above Assumption, (1) implies that a variable is active if and only if its derivative is negative; (2) implies that every configuration is relevant to the Zeno behavior; (3) implies that every variable is relevant to the Zeno behavior; (4) ensures that the hybrid system cannot stay in any configuration indefinitely; and (5) implies that the hybrid system can be forced to exit a configuration at any time before $\left[x_{i} \leq 0\right]$ becomes true. Furthermore, the above assumptions together with the definition of SCC imply that the system can be forced to move instantaneously from any configuration to any other configuration by forcing one or more successive events.

Let us consider a run of a homogeneous constant-rate hybrid system $\mathcal{H}$ initialized at state $x(0)=x_{0}$. We assume that $x_{0}$ is in $\operatorname{int}\left(\mathbb{R}_{+}^{m}\right)$, the interior of $\mathbb{R}_{+}^{m}$. It is convenient to express the system dynamics by

$$
\dot{x}=K \operatorname{In}(t),
$$

where

$$
K=\left[\begin{array}{cccc}
k_{11} & k_{12} & \ldots & k_{1 n} \\
k_{21} & k_{22} & \ldots & k_{2 n} \\
\ldots & & & \\
k_{m 1} & k_{m 2} & \ldots & k_{m n}
\end{array}\right]
$$

Using equation (2), we obtain the state $x(t)$ at $t \geq 0$ as

$$
x(t)=\int_{0}^{t} K \operatorname{In}(\tau) d \tau+x_{0} .
$$

Equation (3) can be rewritten as

$$
x(t)=K \int_{0}^{t} \operatorname{In}(\tau) d \tau+x_{0}=K t \alpha(t)+x_{0},
$$


where $\alpha(t)=\frac{1}{t} \int_{0}^{t} \operatorname{In}(\tau) d \tau=:\left[\alpha_{1}(t) \alpha_{2}(t) \ldots \alpha_{n}(t)\right]^{\prime}$. Note that $\alpha_{i}(t) \geq 0, i=1,2, \ldots, n$, and $\alpha_{1}(t)+\alpha_{2}(t)+\ldots+\alpha_{n}(t)=1$. Thus, $\alpha_{i}(t)$ represents the fraction of the total time (up to time $t$ ), that the system resides in configuration $i ; i=1,2, \ldots, n$. In other words,

$$
\alpha(t) \in \mathcal{A}:=\left\{\alpha \in \mathbb{R}_{+}^{n} \mid \sum_{i=1}^{n} \alpha_{i}=1\right\}
$$

It is readily noted that $x(t)=\int_{0}^{t} K \operatorname{In}(\tau) d \tau+x_{0}$ is also the solution of the following (continuous) constant-rate dynamical system

$$
\left\{\begin{array}{l}
\dot{x}=K \alpha \\
x(0)=x_{0}
\end{array}\right.
$$

for $\alpha=\alpha(t)$. This much simpler "equivalent" system will serve us below to investigate the Zenoness properties of the hybrid system $\mathcal{H}$. In particular, we will show that the existence of Zenoness is closely related to the existence of solutions to the (vector) inequality $K \alpha \geq 0$, $\alpha \in \mathcal{A}$.

We shall make use of the following simple observation.

Lemma 1 Let $\mathcal{H}$ be a homogeneous constant-rate hybrid system satisfying Assumption 1 with initial state $x(0)=x_{0} \in \operatorname{int}\left(\mathbb{R}_{+}^{m}\right)$. Let $x \in \operatorname{int}\left(\mathbb{R}_{+}^{m}\right)$ be any point. Then there exists a run of $\mathcal{H}$ reaching $x$ with a trajectory wholly contained in $\mathbb{R}_{+}^{m}$ if and only if for some $\alpha \in \mathcal{A}$ there exists a solution to system (5) starting at $x_{0}$ and reaching $x$. Moreover, in that case, the time $T$ at which $\mathcal{H}$ reaches $x$ (i.e., $x(T)=x$ ) is the same as the time at which the equivalent system (5) reaches $x$.

\section{Proof}

(Only if) Suppose there exists a state trajectory of $\mathcal{H}$, wholly contained in $\mathbb{R}_{+}^{m}$, starting at $x_{0}$ and reaching $x$ at time $T$; that is, $x(T)=x$. Then, the solution of system (5) starting at $x_{0}$ at time 0 , with the value of $\alpha$ taken as $\alpha(T)$ from Equation 4, will reach the state $x$ at time $T$.

(If) If there exists a trajectory of system (5), for some $\alpha^{*} \in \mathcal{A}$, starting at $x_{0}$ and reaching $x$, then this trajectory is a line segment with endpoints $x_{0}$ and $x$. Assume $x$ is reached at time $T$ (i.e., $x(T)=x$ ). Then any trajectory of HM satisfying $\alpha(T)=\alpha^{*}$ will be a trajectory from $x_{0}$ to $x$. Although not all such trajectories need to be contained in $\mathbb{R}_{+}^{m}$, we will see that there exist trajectories that are. Indeed, since the line segment connecting $x_{0}$ and $x$ is wholly contained in the open set $\operatorname{int}\left(\mathbb{R}_{+}^{m}\right)$, there exists $\epsilon>0$, for which the tube of radius $\epsilon$ around this line segment is also contained in $\operatorname{int}\left(\mathbb{R}_{+}^{m}\right)$. We can construct a run of HM whose trajectory stays within this $\epsilon$-tube (and hence in $\mathbb{R}_{+}^{m}$ ) as follows. We first partition the line segment $\left[x_{0}, x\right]$ into $N$ equal sections. The end points of these sections are denoted by $x^{1}, x^{2}, \ldots, x^{N},\left(x^{N}=x\right)$. Let $t_{i}$ be the time when $x^{i}$ is reached: $x\left(t_{i}\right)=x^{i}, i=1,2, \ldots, N$. Clearly, we can construct a run of HM such that $\alpha\left(t_{i}\right)=\alpha^{*}, i=1,2, \ldots, N$. The trajectory of 
such a run will intersect the line segment $\left[x_{0}, x\right]$ at $x^{1}, x^{2}, \ldots, x^{N}$. By selecting a sufficiently large $N$, we can ensure that the deviation of the trajectory from the line segment $\left[x_{0}, x\right]$ be smaller than $\epsilon$, and hence wholly contained in the $\epsilon$-tube around $\left[x_{0}, x\right]$, concluding the proof.

By investigating the equivalent system (5) instead of the original hybrid system $\mathcal{H}$, we can simplify the problem of determining Zenoness significantly. In particular, we have the following necessary and sufficient condition for strong Zenoness.

Theorem 1 Let $\mathcal{H}$ be a homogeneous constant-rate hybrid system satisfying Assumption 1 with initial state $x(0)=x_{0} \in \operatorname{int}\left(\mathbb{R}_{+}^{m}\right)$. Then $\mathcal{H}$ is strongly Zeno if and only if $K \alpha \geq 0$ has no solutions in $\mathcal{A}$.

\section{Proof}

(If) Assume that $K \alpha \geq 0$ has no solutions in $\mathcal{A}$, but that $\mathcal{H}$ has some non-Zeno run such that for all $t \geq 0$,

$$
x(t)=K \alpha(t) t+x_{0} \in \mathbb{R}_{+}^{m} .
$$

Let $\left\{t_{i}\right\}_{i \in \mathbb{N}}, t_{i+1}>t_{i}$, be an unbounded sequence of times. Then, since $\alpha\left(t_{i}\right) \in \mathcal{A}$ for all $i$, and since $\mathcal{A}$ is compact, the sequence $\alpha\left(t_{i}\right)$ has a convergent subsequence $\alpha\left(t_{j_{i}}\right)$ with limit $\alpha^{*} \in \mathcal{A}$. Let $v=K \alpha^{*}$. Since, by assumption, $K \alpha \geq 0$ has no solutions in $\mathcal{A}$, it follows that $v_{j}<0$ for some $j \in\{1, \ldots, m\}$. Hence, there exists $0<t^{*}<\infty$, such that at least one component of $x(t)=K \alpha^{*} t+x_{0}$ will become negative for all $t>t^{*}$. But then, since $K \alpha t+x_{0}$ is continuous (and in fact linear) function in $\alpha$, we conclude that some component of $x(t)=K \alpha(t) t+x_{0}$ will become negative for finite $t$, contradicting our assumption that a non-Zeno run exists.

(Only if) Suppose there exists $\alpha^{*} \in \mathcal{A}$ such that $K \alpha^{*} \geq 0$. Then for $x_{0} \in \operatorname{int}\left(\mathbb{R}_{+}^{m}\right)$, the trajectory $x(t)=K \alpha^{*} t+x_{0} \in \operatorname{int}\left(\mathbb{R}_{+}^{m}\right)$ for all $t \geq 0$. By Lemma 1 there exists then a run of $\mathcal{H}$ starting at $x_{0}$, which is wholly contained in $\mathbb{R}_{+}^{m}$, in contradiction with the assumption that $\mathcal{H}$ is strongly zeno.

The condition of Theorem 1 (which is the standard feasibility condition for solution of a linear program) can be easily checked. If $K \alpha \geq 0$ has solutions, the hybrid system is not strongly Zeno and there exist switching policies resulting in non-Zeno runs of the system. However, without externally forced switching, the dynamic runs may still be Zeno. We shall discuss the control issues in Section 4.

\subsection{Regular Systems}

Although the problem of finding necessary and sufficient conditions for Zenoness (rather than strong Zenoness) is still open, we can solve the problem for regular systems, which satisfy both Assumption 1 and the following: 
Assumption 2 The number of continuous (state) variables is equal to the number of configurations (that is, $n=m$ ). Furthermore, the rate matrix is of full rank (that is, $\operatorname{rank}(K)=n$ ).

Assumptions 1 and 2 together imply that each state variable is active in exactly one configuration.

To present our results, let us consider all convex cones in $\mathbb{R}^{n}$ rooted at the origin. Denote by

$$
\begin{gathered}
C O N E\left(v_{1}, v_{2}, \ldots, v_{l}\right)=\left\{v \in \mathbb{R}^{n}: \quad v=\beta_{1} v_{1}+\beta_{2} v_{2}+\ldots+\beta_{l} v_{l}\right. \text { for some } \\
\left.\beta_{1} \geq 0, \beta_{2} \geq 0, \ldots, \beta_{l} \geq 0\right\}
\end{gathered}
$$

the convex cone generated by vectors $v_{i} \in \mathbb{R}^{n}, i=1,2, \ldots, l$.

Let $u_{i}=\left[\begin{array}{llll}0 & \ldots & \ldots & \ldots\end{array}\right]^{T}$ be the $n$-vector with 1 in its $i$ th position and 0 elsewhere. Denote

$$
\begin{aligned}
& P O=\operatorname{CONE}\left(u_{1}, u_{2}, \ldots, u_{n}\right) \\
& N E=\operatorname{CONE}\left(-u_{1},-u_{2}, \ldots,-u_{n}\right) .
\end{aligned}
$$

If $\operatorname{rank}\left[v_{1} v_{2} \ldots v_{l}\right]=r$, then the dimension of $\operatorname{CONE}\left(v_{1}, v_{2}, \ldots, v_{l}\right)$ is $r$. Its boundary consists of $r$ surfaces. Each surface is a part of a supporting hyperplane, generated by some set of $r-1$ independent vectors in $\left\{v_{1}, v_{2}, \ldots, v_{l}\right\}$.

Lemma 2 Let $C_{1}$ and $C_{2}$ be two convex cones. If the cone $C_{2}$ intersects the surfaces (boundary) of $C_{1}$ only at the origin, then either $C_{2}$ is contained in $C_{1}$, or $C_{2}$ is contained in the complement of $C_{1}$.

\section{Proof}

Elementary.

Denote the column vectors of $K$ by $k_{i}: K=\left[k_{1} k_{2} \ldots k_{n}\right]$.

Lemma 3 Under Assumption 2, the surfaces of $C O N E\left(k_{1}, k_{2}, \ldots, k_{n}\right)$ and $N E$ intersect only at the origin.

\section{Proof}

Under Assumption 2, the matrix $\left[k_{i_{1}} k_{i_{2}} \ldots k_{i_{n-1}}\right]$ consisting of any $n-1$ columns of $K$, $k_{i_{1}}, k_{i_{2}}, \ldots, k_{i_{n-1}}$, has at least one row all of whose elements are nonnegative. Therefore, the surface generated by the vectors $k_{i_{1}}, k_{i_{2}}, \ldots, k_{i_{n-1}}$ intersects with $N E$ only at the origin.

Lemma 4 Under Assumption 2, $K \alpha \geq 0$ has no solution in $\mathcal{A}$ if $K \alpha<0$ has a solution in $\mathcal{A}$. 


\section{Proof}

By Lemmas 2 and 3, NE is either contained in $C O N E\left(k_{1}, k_{2}, \ldots, k_{n}\right)$, or is contained in the complement of $C O N E\left(k_{1}, k_{2}, \ldots, k_{n}\right)$.

Suppose $K \alpha<0$ has a solution in $\mathcal{A}$. This means that $C O N E\left(k_{1}, k_{2}, \ldots, k_{n}\right) \cap N E \neq\{0\}$. Therefore, $N E$ is contained in $\operatorname{CONE}\left(k_{1}, k_{2}, \ldots, k_{n}\right)$ and hence $\operatorname{CONE}\left(k_{1}, k_{2}, \ldots, k_{n}\right) \cap$ $P O=\{0\}$. Because $K$ is of full rank, $K \alpha \geq 0$ has no solution in $\mathcal{A}$.

With these three lemmas, we can prove the following theorem that gives a necessary and sufficient condition for Zenoness of regular systems.

Theorem 2 Under Assumptions 1 and 2, a homogeneous constant-rate hybrid system $\mathcal{H}$ is Zeno if and only if $K \alpha \geq 0$ has no solution in $\mathcal{A}$.

\section{Proof}

If $K \alpha \geq 0$ has no solution in $\mathcal{A}$, then by Theorem $1, \mathcal{H}$ is strongly Zeno and hence Zeno. If $\mathcal{H}$ is Zeno, then it has a Zeno run. Let $\alpha_{z} \in \mathcal{A}$ be associated with that run. Clearly $K \alpha_{z}<0$. By Lemma 4 , the system of inequalities $K \alpha \geq 0$ has no solution in $\mathcal{A}$.

Note that for systems satisfying both Assumption 1 and Assumption 2, Zenoness and strong Zenoness are equivalent; that is, there exists a Zeno run of a system if and only if all its runs are Zeno. Also note that for systems satisfying Assumption 1 but not Assumption 2 , no conclusion can be drawn just from the existence of solutions in $\mathcal{A}$ to the inequality $K \alpha \geq 0$, as to whether the system is Zeno or not. In the next subsection, we shall provide illustrative examples to demonstrate different aspects of Zenoness for such cases.

\subsection{Illustrative Examples}

Zeno behaviors have a complex nature even for systems satisfying Assumption 1 (but not Assumption 2) as we will illustrate by the following examples. Note that when the conditions of Theorem 1 or Theorem 2 are satisfied, then the results are independent of the initial conditions and the exact layout of connections between configurations. However, when these conditions are not satisfied, a dynamic run may or may not be Zeno depending on the initial conditions and on the exact layout of connections and guards between configurations. This is illustrated in Examples 2 and 3.

Example 2 This example shows a hybrid system in which certain dynamic runs are Zeno and others are not, depending on the initial condition. The system is shown in Figure 2.

This system satisfies Assumption 1 but not Assumption 2 (and hence is not regular), since the second configuration has two active variables. Notice further that $K \alpha \geq 0$ has solutions

in $\mathcal{A}$ and $K \alpha<0$ has no solutions in $\mathcal{A}$ for this system. To understand the dynamic behavior 


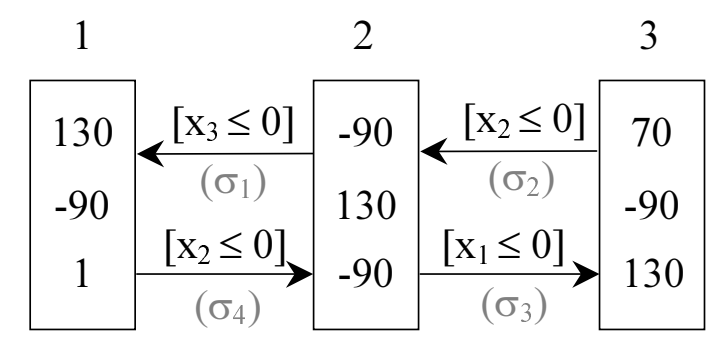

Figure 2: A system where Zenoness depends on the initial state

of this system, observe that the loop consisting of configurations 1 and 2 (denoted by $1 \leftrightarrow 2$ ) has active variables $x_{2}$ and $x_{3}$. The submatrix corresponding to these variables is

$$
K_{s u b}^{L}=\left[\begin{array}{rr}
-90 & 130 \\
1 & -90
\end{array}\right] \text {, }
$$

and represents a Zeno regular hybrid system; that it, $K_{\text {sub }}^{L}$ satisfies Assumption 2 and $K_{\text {sub }}^{L} \alpha \geq 0$ has no solutions in $\mathcal{A}_{\text {sub }}^{L}:=\left\{\alpha_{2}, \alpha_{3} \mid \alpha_{2} \geq 0, \alpha_{3} \geq 0, \alpha_{2}+\alpha_{3}=1\right\}$. Thus, if a dynamic run is "trapped" in the loop $1 \leftrightarrow 2$, Zeno behavior must occur.

On the other hand, the loop $2 \leftrightarrow 3$ consisting of configurations 2 and 3 , has active variables 1 and 2 with associated submatrix

$$
K_{\text {sub }}^{R}=\left[\begin{array}{rr}
-90 & 70 \\
130 & -90
\end{array}\right]
$$

which represents a non-Zeno regular hybrid system $\left(K_{\text {sub }}^{R} \alpha \geq 0\right.$ has solutions in $\left.\mathcal{A}_{\text {sub }}^{R}\right)$. Hence, if a dynamic run is "trapped" in the loop $2 \leftrightarrow 3$, it will be non-Zeno.

One can see that the system of Figure 2 will be trapped in one of the two loops after a number of initial transitions. Suppose that the initial configuration is 1 . When $x_{2}=0$, a transition takes the system to configuration 2. Now suppose $x_{3}$ hits its guard before $x_{1}$ (i.e., $x_{3}=0$ is reached while $x_{1}>0$ ) and the system switches back to configuration 1 , where the rate of $x_{1}$ is greater than the rate of $x_{3}$. After a while, the transition to configuration 2 takes place again, where $x_{1}$ and $x_{3}$ have the same negative rate, and therefore $x_{3}$ will again become zero before $x_{1}$, forcing the system back to configuration 1 , and so on.

Thus, the behavior of the system is given by the matrix $K_{s u b}^{L}$, corresponding to $x_{2}$ and $x_{3}$ in configurations 1 and 2. On the other hand, if after the first transition, $x_{1}$ becomes zero before $x_{3}$, a similar argument shows that the behavior depends only on the matrix $K_{s u b}^{R}$ corresponding to $x_{1}$ and $x_{2}$ in configurations 2 and 3 . Therefore, we conclude that the run will or will not be Zeno, depending on the initial state. A simple calculation shows that, for $q_{0}=1$, the run is Zeno if $x_{0_{1}}>x_{0_{3}}-(129 / 90) x_{0_{2}}$, and it is non-Zeno if $x_{0_{1}}<x_{0_{3}}-(129 / 90) x_{0_{2}}$. In the case of equality, then after the first transition (from configuration 1 to configuration 2 ), both variables $x_{1}$ and $x_{3}$ become zero in configuration 2 at the same instant, and the system chooses its next configuration (either 1 or 3 ) non-deterministically, thereby becoming 
Zeno if it switches to configuration 1 and non-Zeno if it switches to configuration 3. Two sample runs that demonstrate Zeno and non-Zeno behaviors of this system are shown in Figure 3.

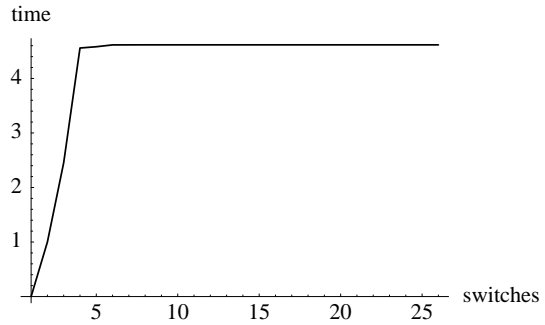

(a) Zeno run: Infinite switches confined to bounded time

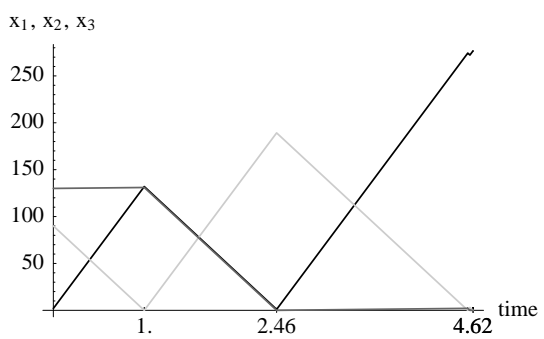

(c) Zeno Run: The state variables

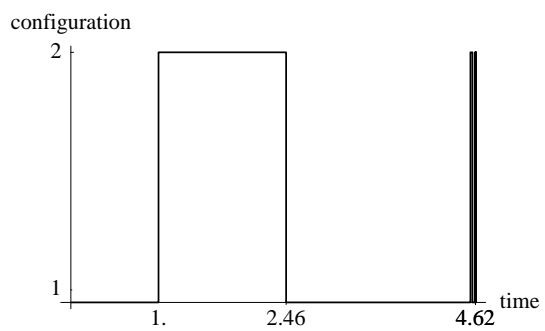

(e) Zeno run: Switching pattern

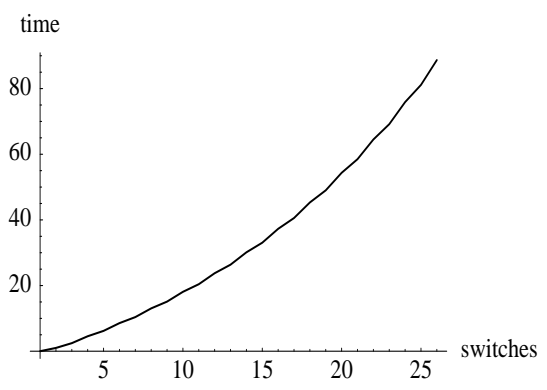

(b) Non-Zeno run: Switches take place in unbounded time

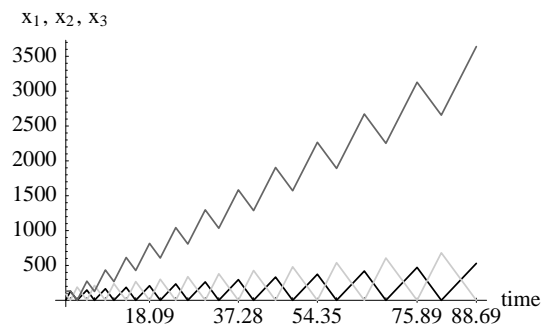

(d) Non-Zeno run: The state variables

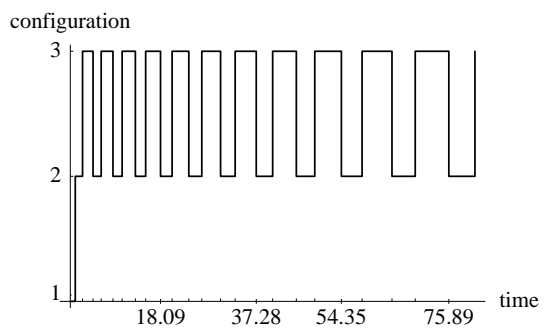

(f) Non-Zeno run: Switching pattern

Figure 3: Representative Runs

(A) Zeno Run: $x_{0}=[2,90,130], q_{0}=1$; (B) Non-Zeno Run: $x_{0}=[1,90,131], q_{0}=1$

Example 3 This example shows two systems with identical configurations and dynamics (i.e., with the same rate matrix K), as well as identical invariants, that differ in their connection-layouts between configurations. One of these layouts is shown in Figure 4(a) and yields a non-Zeno system, while the other layout is shown in Figure 5(a) and yields a Zeno system. 


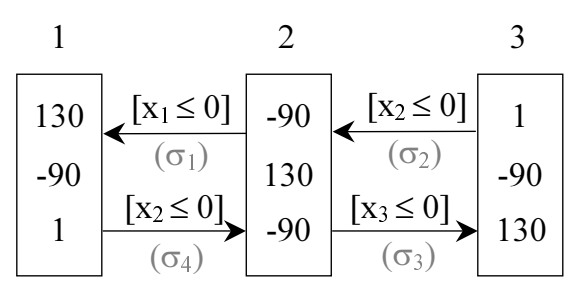

(a) The hybrid system

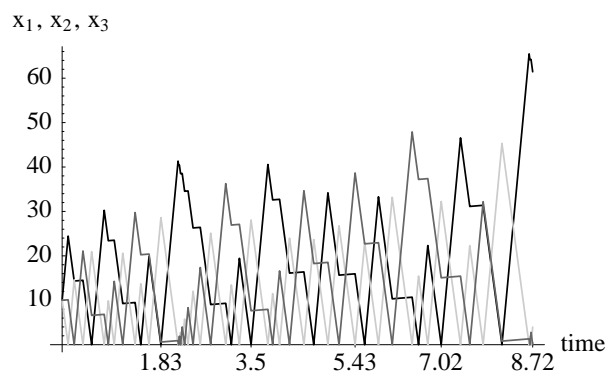

(c) The state variables

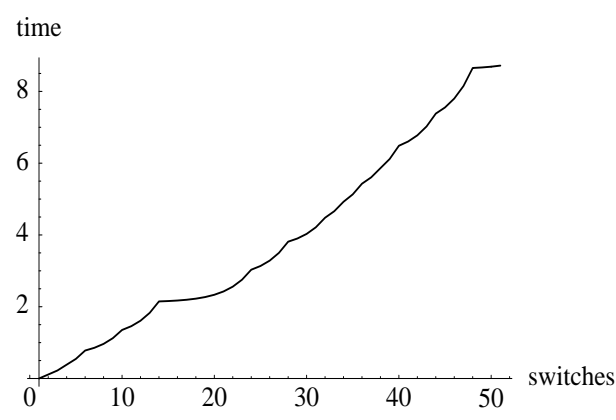

(b) Switches occur in unbounded time

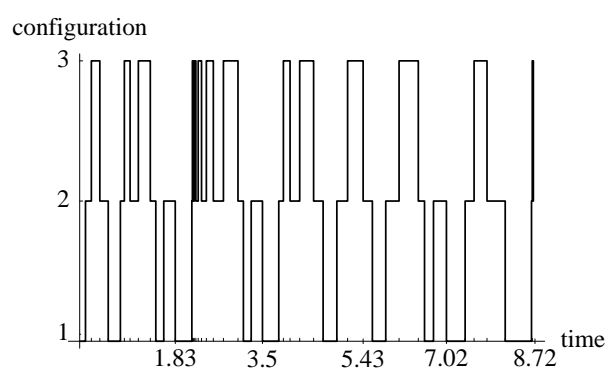

(d) The switching

Figure 4: Non-Zeno layout

Notice that when the system of Figure 4(a) is in configuration 2, the condition $\left[x_{1} \leq 0\right]$ triggers a transition to configuration 1 , where $\dot{x_{1}}=130$. On the other hand, in the system of Figure $5(\mathrm{a})$, the condition $\left[x_{1} \leq 0\right]$ triggers a transition to configuration 3 , where $\dot{x_{1}}=1$ (which is much smaller than 130).

Let us compare the individual loops in the two systems. For the system in Figure 4(a), the loop $1 \leftrightarrow 2$ has active variables $x_{1}$ and $x_{2}$, whose rate matrix corresponds to non-Zeno behavior. Similarly, the loop $2 \leftrightarrow 3$ has active variables $x_{2}$ and $x_{3}$, whose rate matrix also corresponds to non-Zeno behavior. On the other hand, in the system shown in Figure 5(a), the loop $1 \leftrightarrow 2$ has active variables $x_{2}$ and $x_{3}$ and the loop $2 \leftrightarrow 3$ has active variables $x_{1}$ and $x_{2}$. The rate matrices of both these loops correspond to Zeno behaviors.

Using reasoning similar to that in Example 2, one can see that the system shown in Figure 5(a) will be trapped in one of the two loops following some initial transitions. In either loop, the system is Zeno.

On the other hand, an analysis of the system shown in Figure 4(a) reveals that the system is never trapped in one of the two loops. Still, in spite of the non-Zeno run exhibited in Figure 4, no general conclusion can be drawn regarding the Zenoness of the system . (Recall Example 1, where the system had two non-Zeno loops yet the system was still Zeno.) 


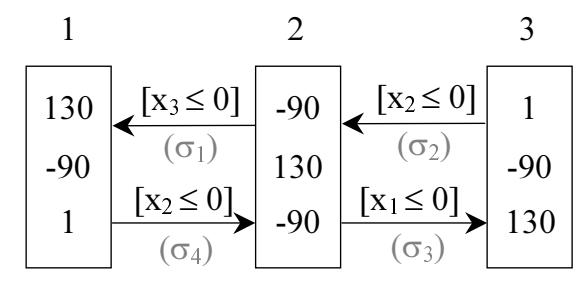

(a) The hybrid system

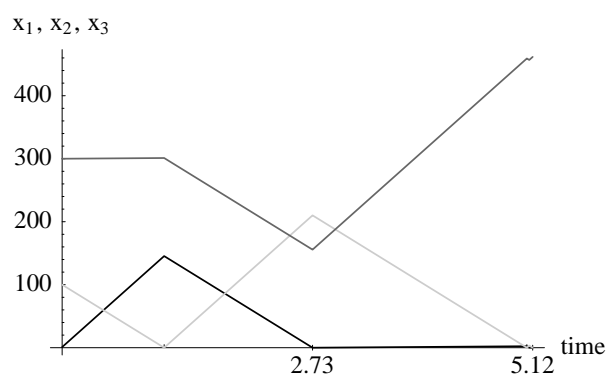

(c) The state variables; the Zeno point is not zero

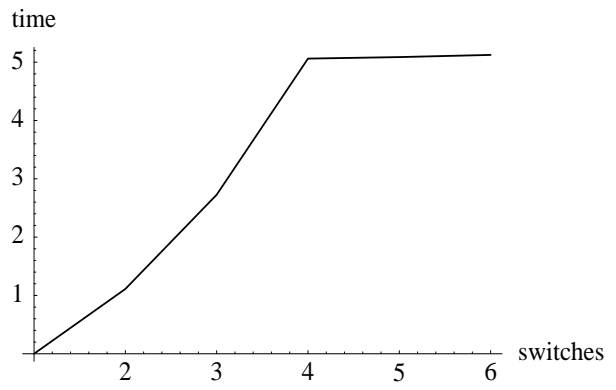

(b) Infinite switches occur in bounded time

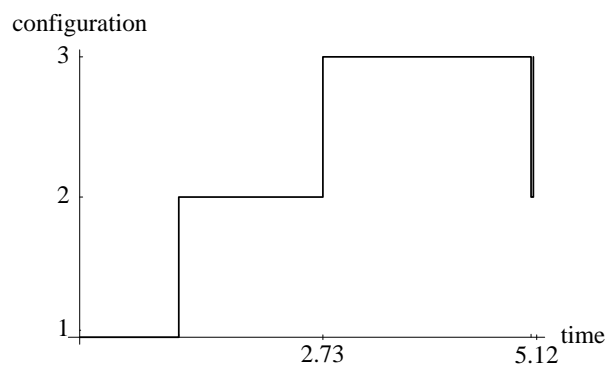

(d) The switching

Figure 5: Zeno layout for the same K

Example 4 This example shows that even for a Zeno system that has only one loop (and hence only one switching sequence), there may exist non-Zeno runs when switched properly.

The system is shown in Figure 6(a). Its dynamic run (i.e., when switched by the guards becoming true) is Zeno as shown in Figure 6(b)- Figure 6(d). However, $K \alpha \geq 0$ has solutions in $\mathcal{A}$. For example, one solution is $\alpha^{*}=[0.125,0.125,0.5,0.25]^{T}$. Therefore, if the system is switched to remain in the proximity of the line emanating from $x_{0}$ in the direction of $\alpha^{*}$ (as discussed in the proof of Lemma 1), the run will be non-Zeno.

\section{Zenoness in Controlled Hybrid Systems}

In the previous section, we have examined various conditions for Zeno behaviors and derived necessary and sufficient conditions for Zenoness in constant-rate hybrid systems. In this section, we shall use these results to investigate the existence and synthesis of controllers for such systems.

A controller $C$ of a hybrid system $\mathcal{H}$ can be thought of as another hybrid system that runs in parallel with $\mathcal{H}$. The controlled system (also called closed-loop system) is the composite of the two running in parallel and is denoted by $\mathcal{H} \| \mathcal{C}$. We assume that $C$ and $\mathcal{H}$ interact by event synchronization only. That is, $C$ controls $\mathcal{H}$ only by triggering (event) transitions and 


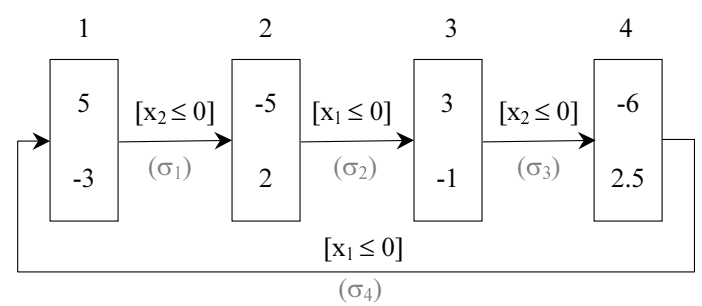

(a) The hybrid system

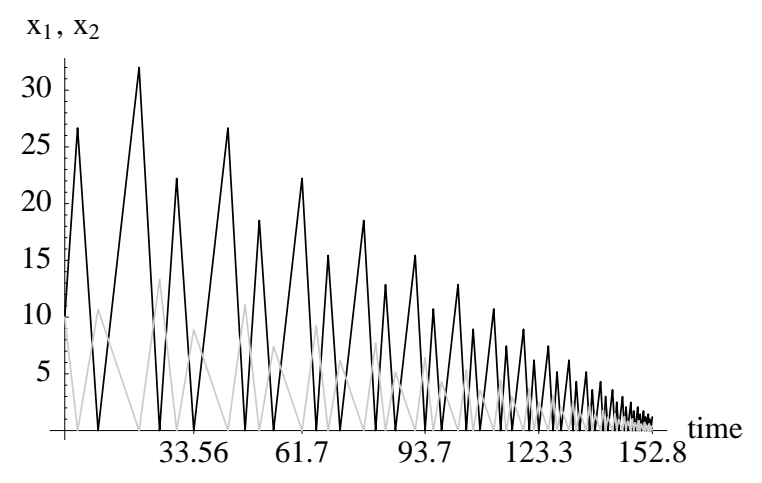

(c) The state variables

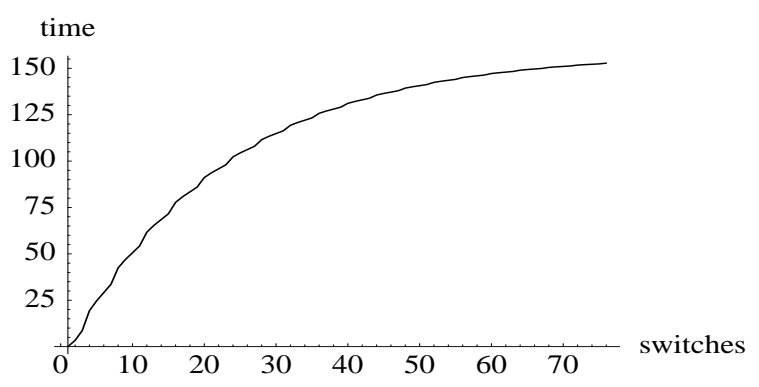

(b) Infinite switches occur in bounded time

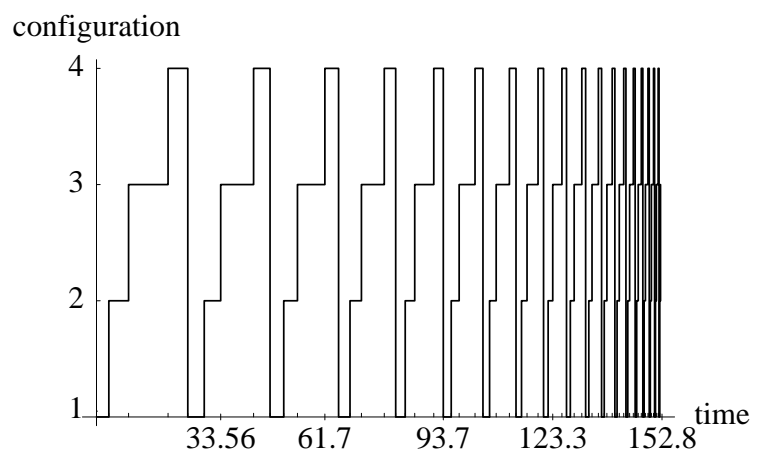

(d) The switching

Figure 6: A Zeno system, for which $K \alpha \geq 0$ has solutions in $\mathcal{A}$

does not interfere otherwise with the continuous dynamics of $\mathcal{H}[12]$.

Controllers are used to ensure the satisfaction of safety [3] [6] [12] [15] [19], liveness [13] and optimality specifications of systems. A safety specification is a state-invariance constraint that specifies a 'legal' region of operation in which the system must remain at all time. A safety controller is aimed at ensuring that the system never leave the specified legal region. Various algorithms have been proposed in the literature for synthesis of safety controllers for hybrid systems. Essentially, all these are iterative "layer peeling" algorithms that employ the following basic approach: Let $L$ and $I$ denote the legal and illegal regions, respectively, of the operating space. Then at the $i^{\text {th }}$ iteration, the algorithm computes the set of all initial states (or, region in the operating space) $S_{i}$, from which there exists no control policy under which the safety requirement can be satisfied for more than $i-1$ discrete (switching) steps. Consequently, when starting in any state of $S_{i}$, it will take, for any control policy, at most $i$ steps before the system state will enter $I$. The $(i+1)^{t h}$ iteration of the algorithm consists of computing the set $S^{i+1} \subseteq L-S_{i}$ of states from which, in at most one step, the system will be forced to enter $I \bigcup S_{i}$, so that $S_{i+1}=S^{i+1} \bigcup S_{i}$. The algorithm terminates at step $i$ either if $\left(q_{0}, x_{0}\right) \in S_{i}$ and a safety controller does not exist, or if a fixed point is reached; that is, $S_{i+1}=S_{i}\left(=: S^{*}\right)$. There is, of course, also the possibility that the algorithm never terminates and no conclusion can be drawn at all. In case, the algorithm 
terminates finitely, and a fixed point is reached, a safety controller exists and the system can be controlled to satisfy the safety specification indefinitely, provided the system is non-Zeno $[10,12]$. We then say that the controlled system is viable $[10,12]$. Moreover, the controller $C$ is obtained from the synthesis algorithm as the hybrid system that forces configuration transitions in the controlled system when the boundary of $L-S^{*}$ is reached, thereby avoiding entrance into the illegal region $I$. Such a controller, is then minimally interventive [10, 12].

The situation gets to be complicated when the system to be controlled is Zeno. In that case, a controller may or may not exist even if the synthesis algorithm terminates finitely. Moreover, even if a safety controller for a Zeno system exists, there may not exist a minimally interventive one as will be seen below. Finally, as mentioned above, there remains the case in which the algorithm does not converge finitely and the existence of a safety controller cannot be resolved algorithmically.

In this section we discuss the issue of controller existence and synthesis in some detail. For the purpose of our discussions, we shall assume that the system to be controlled is a constant-rate hybrid system with rate matrix $K$ that satisfies Assumption 1. In particular, Assumption 1 implies that all dynamic transitions can be preempted by controls (i.e., the transitions can be triggered by the controller at or before the corresponding guards become true). Furthermore, any configuration can be reached instantaneously from any other by a suitable sequence of controlled transitions.

For systems that satisfy the above conditions, the standard synthesis algorithm terminates in one iteration and generates a controller $C$, that switches configurations whenever the boundary of the legal region is reached; namely, whenever the active variable becomes zero.

The obtained controller $C$ may or may not be viable, depending on whether the closedloop system $\mathcal{H} \| \mathcal{C}$ is Zeno or not. For systems that satisfy Assumption 1, we can reach the following conclusions from the results of the previous section.

1. If $K \alpha \geq 0$ has no solution in $\mathcal{A}$, then by Theorem 1 , any run of $\mathcal{H}$ is Zeno. Therefore $\mathcal{H} \| \mathcal{C}$ is Zeno. In fact, no viable controller for $\mathcal{H}$ exists and there is no point in even trying to synthesize a controller.

2. If $\mathcal{H}$ also satisfies Assumption 2 and hence is regular, and if $K \alpha \geq 0$ has a solution in $\mathcal{A}$, then by Theorem 2, no run of $\mathcal{H}$ is Zeno. Therefore $\mathcal{H} \| \mathcal{C}$ is non-Zeno and $C$ is the minimally interventive safety controller.

3. If the hybrid system $\mathcal{H}$ satisfies Assumption 1 but not assumption 2 (and hence is not regular), and $K \alpha \geq 0$ has a solution in $\mathcal{A}$, then the behavior of the synthesis algorithm and properties of the controller cannot be determined from the results of the previous section, and some further examination is required.

In Case 3 , since $K \alpha \geq 0$ has a solution in $\mathcal{A}$, a non-Zeno safety controller exists. However, it may not be obtainable by the standard algorithmic approach. Moreover, while a controller 
exists, there is no guarantee that there exists a minimally interventive one. Indeed, as illustrated in Example 4, if $\alpha^{*}$ is a solution to $K \alpha \geq 0$, a controller can be obtained by switching configurations to remain in the proximity of the ray emanating from the initial state $x_{0}$ in the direction of $\alpha^{*}$, as was discussed earlier. However, while such a controller is viable and guarantees safety, it cannot be synthesized using the standard algorithmic approach. To see this, let us return to Example 4. Note that the controller $C$ synthesized by the standard approach switches on the boundary of $\mathbb{R}_{+}^{m}$, and the closed-loop system which is represented by Figure 6(a), is Zeno. This is also an example in which a minimally interventive safety controller does not exist. Indeed, consider a controller that operates in two phases. In the first phase the controller switches configurations on the boundary of $\mathbb{R}_{+}^{m}$ for a finite but arbitrarily large number of times. It holds the system in $\mathbb{R}_{+}^{m}$ in a minimally interventive way, while allowing the system to approach the Zeno point. The second phase starts at a point $x \in \operatorname{int}\left(\mathbb{R}_{+}^{m}\right)$ that the system reaches in the first switching phase, and the controller begins to switch configurations so as to remain in $\mathbb{R}_{+}^{m}$ close to line emanating from $x$ in the direction of $\alpha^{*}=[0.125,0.125,0.5,0.25]^{T}$. The result is a viable and non-Zeno safety controller. This procedure enables us to design a controller that allows the system to get arbitrarily close to the Zeno point (by switching on the boundary in a minimally interventive way) and then drive it away from the Zeno point (by switching "along" $\alpha^{*}$ ). Hence, for any safety controller of the type synthesized, there exists another controller that allows the system in the first phase to get yet closer to the Zeno point before driving it away. It follows that for any safety controller there is another safety controller that is less interventive (by staying longer in phase one). It follows that a minimally interventive controller does not exist. However, this is equivalent to the fact that a maximal control-invariant set does not exist, disproving the conjecture made in [16] (Proposition 3) that if a viable controller exists, then a uniqe maximal control-invariant set exists as well.

Since it has been understood for some time that system Zenoness is an impediment to controller synthesis, various ways have been sought to prevent and bypass its possible occurrence [14]. In particular, it has been argued that Zenoness is a modelling artifice and real physical systems cannot switch configurations at an arbitrarily high rate. Thus, various model "regularization" methods have been suggested in the literature that are aimed at forcing delays between successive configuration switches, thereby preventing Zenoness from occurring. It is interesting to examine what the actual effect is of model regularization and Zenoness elimination on the controller synthesis results. To this end, let us reexamine each of the three cases discussed above:

1. Clearly, in case 1 , the introduction of switching delays will not help, since in this case all runs are Zeno and obviously no safety controller exists, regardless as to whether or not delays are permitted. The iterative synthesis procedure will either fail to converge or will decide finitely that a controller with the specified delay does not exist. However, there is no indication whether a controller with a smaller delay exists or not. One may 
falsely hope that it does!

2. In Case 2, the minimally interventive controller exists without a delay specification. Hence, the standard algorithm terminates finitely, with a viable controller design. However, a controller may not exist for a specified minimal delay and a given initial condition. Therefore, one may falsely conclude that a controller does not exist.

3. Case 3 is the only case in which the introducion of a delay may help. This can be seen from examination of Example 3. Synthesis without a delay results in a controller that switches nondeterministically either according to Figure 4(a) or according to Figure 5(a). Such switching may (but does not need to) produce a Zeno run. If the system is "regularized", for example by introduction of a delay, the controller synthesis algorithm will produce the correct switching pattern as shown in Figure 4(a). The introduction of the delay in the algorithm is not cost-free because we know that a non-Zeno safety controller without specified delay actually exists and can be found by the analysis presented in the present paper, which can be employed for selecting the preferred switching pattern. The introduction of a delay in systems that fall into 'Case 3' may have other undesirable effects as shown in Example 4. For this Zeno system the standard synthesis algorithm with delay does not converge finitely and hence does not terminate. However, we know that a safety controller exists (although not a minimally interventive one).

It is clear from the above discussion that while the introduction of a delay (or other "regularization" procedure) prevents the system from becoming Zeno, it is not an effective method for solving the safety controller design problem for Zeno (or non-Zeno) hybrid systems.

\section{Extensions to Bounded-Rate Hybrid Systems}

In this section, we extend the results of Section 3 to bounded-rate hybrid systems. In a bounded-rate system, the dynamic behavior is given by

$$
\dot{x}_{i} \in\left[k_{i j}^{L}, k_{i j}^{U}\right], i=1,2, \ldots, m, j=1,2, \ldots, n .
$$

where $k_{i j}^{L}$ and $k_{i j}^{U}$ are the lower and upper bounds of the rates. By bounded rate we mean that all that is assumed about the dynamics is that the rate can take any (possibly time varying) value in the specified range (subject, of course, to standard integrability conditions). Let us define lower and upper rate-bound matrices

$$
K^{L}:=\left[\begin{array}{cccc}
k_{11}^{L} & k_{12}^{L} & \ldots & k_{1 n}^{L} \\
k_{21}^{L} & k_{22}^{L} & \ldots & k_{2 n}^{L} \\
\ldots & & & \\
k_{m 1}^{L} & k_{m 2}^{L} & \ldots & k_{m n}^{L}
\end{array}\right]
$$




$$
K^{U}:=\left[\begin{array}{cccc}
k_{11}^{U} & k_{12}^{U} & \ldots & k_{1 n}^{U} \\
k_{21}^{U} & k_{22}^{U} & \ldots & k_{2 n}^{U} \\
\ldots & & & \\
k_{m 1}^{U} & k_{m 2}^{U} & \ldots & k_{m n}^{U}
\end{array}\right]
$$

For a bounded-rate system $\mathcal{H}^{o}$, we define lower-rate system $\mathcal{H}^{\mathcal{L}}$ and upper-rate system $\mathcal{H}^{\mathcal{U}}$ as the constant-rate hybrid systems obtained from $\mathcal{H}^{\circ}$ by replacing the continuous dynamics with constant-rate dynamics given by $K^{L}$ and $K^{U}$, respectively. Our objective is to show that we can investigate Zenoness of $\mathcal{H}^{o}$ by investigating Zenoness of $\mathcal{H}^{\mathcal{L}}$ and $\mathcal{H}^{\mathcal{U}}$. To this end, let us first prove the following lemma.

Lemma 5 Assume $\mathcal{H}^{\mathcal{L}}$ and $\mathcal{H}^{\mathcal{U}}$ both satisfy Assumption 1. (1) If $\mathcal{H}^{o}$ has a non-Zeno run then $\mathcal{H}^{\mathcal{U}}$ has a non-Zeno run. (2) If $\mathcal{H}^{\mathcal{L}}$ has a non-Zeno run then $\mathcal{H}^{o}$ has a non-Zeno run.

\section{Proof}

We prove only (1) because the proof of (2) is similar. Let

$$
r^{o}=q_{0} \stackrel{e_{1}, t_{1}}{\longrightarrow} q_{1} \stackrel{e_{2}, t_{2}}{\longrightarrow} q_{2} \stackrel{e_{3}, t_{3}}{\longrightarrow} \ldots
$$

be a non-Zeno legal run of $\mathcal{H}^{o}$. Let

$$
r^{U}=q_{0} \stackrel{e_{1}, t_{1}}{\longrightarrow} q_{1} \stackrel{e_{2}, t_{2}}{\longrightarrow} q_{2} \stackrel{e_{3}, t_{3}}{\longrightarrow} \ldots
$$

be a run of $\mathcal{H}^{\mathcal{U}}$ that switches at exactly the same times and to exactly the same configurations as $r^{o}$. We need to show that $r^{U}$ is a legal run of $\mathcal{H}^{\mathcal{U}}$. To this end, we shall see that $x^{U}(t) \geq x(t) \geq 0$ for all $t$. This can be done inductively as follows: Initially, $x^{U}(0)=x(0)=$ $x_{0}>0$. Let us suppose that upon entry to $q_{j}, x^{U}\left(t_{i}\right) \geq x\left(t_{i}\right) \geq 0$. Since at configuration $j$, $\dot{x}^{U}=k_{j}^{U} \geq \dot{x}$, it follows that $x^{U}(t) \geq x(t) \geq 0$ for $t \in\left[t_{i}, t_{i+1}\right]$ (where the inequality $x(t) \geq 0$ follows from the assumption that $r^{o}$ is a legal run).

We can now give a sufficient condition for strong Zenoness of bounded rate hybrid systems as follows.

Theorem 3 Assume $\mathcal{H}^{\mathcal{U}}$ satisfies Assumption 1. If $K^{U} \alpha \geq 0$ has no solutions in $\mathcal{A}$, then $\mathcal{H}^{o}$ is strongly Zeno.

\section{Proof}

Suppose that $\mathcal{H}^{\circ}$ is not strongly Zeno, i.e., there exists a non-Zeno run of $\mathcal{H}^{\circ}$. By Lemma 5 , there exists a non-Zeno run of $\mathcal{H}^{\mathcal{U}}$. This means that $\mathcal{H}^{\mathcal{U}}$ is not strongly Zeno. By Theorem $1, K^{U} \alpha \geq 0$ has a solution in $\mathcal{A}$, a contradiction.

Next, we give a necessary condition for strong Zennoness. 
Theorem 4 Assume $\mathcal{H}^{\mathcal{L}}$ satisfies Assumption 1 , if $\mathcal{H}^{o}$ is strongly Zeno, then $K^{L} \alpha \geq 0$ has no solutions in $\mathcal{A}$.

\section{Proof}

Suppose that $K^{L} \alpha \geq 0$ has a solution in $\mathcal{A}$. By Theorem $1, \mathcal{H}^{\mathcal{L}}$ is not strongly Zeno, i.e., there exists a non-Zeno run of $\mathcal{H}^{\mathcal{L}}$. By Lemma 5 , there exists a non-Zeno run of $\mathcal{H}^{o}$, which means that $\mathcal{H}^{o}$ is not strongly Zeno, a contradiction.

The above results deal with strong Zenoness. We now consider Zenoness in regular bounded rate hybrid systems, namely, systems in which $\mathcal{H}^{\mathcal{L}}$ and $\mathcal{H}^{\mathcal{U}}$ both satisfy Assumptions 1 and 2 .

Theorem 5 Assume $\mathcal{H}^{\mathcal{L}}$ satisfies Assumptions 1 and 2. If $K^{L} \alpha \geq 0$ has a solution in $\mathcal{A}$, then $\mathcal{H}^{o}$ is not Zeno.

\section{Proof}

Suppose that $\mathcal{H}^{\circ}$ is Zeno. Let

$$
r^{o}=q_{0} \stackrel{e_{1}, t_{1}}{\longrightarrow} q_{1} \stackrel{e_{2}, t_{2}}{\longrightarrow} q_{2} \stackrel{e_{3}, t_{3}}{\longrightarrow} \ldots
$$

be a Zeno run of $\mathcal{H}^{o}$. This run will reach the Zeno point $(x=0)$ at some finite time $t_{Z}$. Let

$$
r^{L}=q_{0} \stackrel{e_{1}, t_{1}}{\longrightarrow} q_{1} \stackrel{e_{2}, t_{2}}{\longrightarrow} q_{2} \stackrel{e_{3}, t_{3}}{\longrightarrow} \ldots
$$

be a run of $\mathcal{H}^{\mathcal{L}}$ that switches at exactly the same times and to exactly the same configurations as $r^{o}$. (We assume, for the purpose of the proof, that in $\mathcal{H}^{\mathcal{L}}$ the guards $\left[x_{i} \leq 0\right]$ are not in effect and the legal constraints $\left[x_{i} \geq 0\right], i=1,2, \ldots, n$, are not binding.)

It can then be shown, in similar fashion to the proof of Lemma 5 , that $x^{L}(t) \leq x(t)$ for all $t \leq t_{Z}$. In particular, $x^{L}\left(t_{Z}\right)=K^{L} \alpha\left(t_{Z}\right) t_{Z}+x_{0} \leq 0$, where $\alpha\left(t_{Z}\right) \in \mathcal{A}$. This implies that $K^{L} \alpha\left(t_{Z}\right) \leq-x_{0} / t_{Z}<0$, or that $K^{L} \alpha<0$ has a solution in $\mathcal{A}$. By Lemma 4 , it then follows that $K \alpha \geq 0$ has no solution in $\mathcal{A}$, a contradiction.

Example 5 This simple example shows that the above conditions cannot be extended by much. Consider the bounded-rate hybrid system that has two configurations in one loop, with

$$
K^{L}=\left[\begin{array}{rr}
1 & -5 \\
-1 & 1
\end{array}\right] \quad \text { and } K^{U}=\left[\begin{array}{rr}
5 & -1 \\
-1 & 1
\end{array}\right]
$$

One can see that $K^{L} \alpha \geq 0$ has no solution in $\mathcal{A}$, while $K^{U} \alpha \geq 0$ has solutions in $\mathcal{A}$. The behavior of this system is hard to predict from knowing only $K^{L}$ and $K^{U}$. If the actual rates are given by $K^{L}$, then the system is strongly Zeno while, if the rates are given by $K^{U}$, then the system is non-Zeno. 


\section{Zenoness in Systems with Nonlinear Dynamics}

In the previous sections we investigated the occurrence of Zenoness in constant-rate and bounded-rate hybrid systems. The results obtained there can be extended to a more general class of systems. In particular, they can be extended to systems with nonlinear dynamic that can be approximated by constant-rate systems in the vicinity of the Zeno point. Similar approximations of hybrid systems with nonlinear dynamics have been employed in [8], [21], where questions related to reachability properties have been studied.

We consider systems in which the dynamics of variable $i$ in configuration $j$ is given by

$$
\dot{x}_{i}=g_{i j}\left(x_{1}, \ldots, x_{m}\right)
$$

where the $g_{i j}\left(x_{1}, \ldots, x_{m}\right), i=1, \ldots, m ; j=1, \ldots, n$, are Lipschitz continuous functions of $x_{1}, \ldots, x_{m}$. As before, let us assume that the legal region is the nonegative orthant: $x_{i} \geq 0$, $i=1, \ldots, m$. We shall focus attention on the dynamic behavior of the system in the vicinity of the Zeno point $x=0$.

Taking the Taylor expansion of $g_{i j}, i=1,2, \ldots, m ; j=1,2, \ldots, n$, around $x=0$, we obtain,

$$
\dot{x}_{i} \cong g_{i j}(0, \ldots, 0)+\frac{\partial g_{i j}}{\partial x_{1}}(0, \ldots, 0) x_{1}+\ldots+\frac{\partial g_{i j}}{\partial x_{m}}(0, \ldots, 0) x_{m}+\ldots
$$

which, if $g_{i j}(0, \ldots, 0) \neq 0$, behaves, for $x$ sufficiently close to 0 , like

$$
\dot{x}_{i}=g_{i j}(0, \ldots, 0) \text {. }
$$

But this latter equation represents a constant-rate hybrid system and hence the Zenoness of the nonlinear hybrid system is determined by its constant-rate approximation near the origin. Thus, we can apply the results of the previous sections to investigate Zenoness of a fairly large class of nonlinear hybrid systems. Specifically, to nonlinear systems for which $g_{i j}(0, \ldots, 0) \neq 0$ for all $i$ and $j$. For these systems, if the approximate constant-rate system is non-Zeno, then the original nonlinear system is also non-Zeno. On the other hand, if the approximate system is Zeno or strongly Zeno, then the original nonlinear system will have a Zeno run in the vicinity of the Zeno point $x=0$. However, it does not follow that every run of the nonlinear system will reach the vicinity of the Zeno point and hence the nonlinear system may not be strongly Zeno, even when the approximate system is strongly Zeno.

\section{Conclusion}

In this paper we studied various issues concerning the possible existence of Zeno behaviors in hybrid systems and the related question of existence of safety controllers that satisfy specified state invariance constraints. We first focused our attention on constant-rate hybrid systems, and showed that the existence of Zeno behaviors can be examined by checking for existence 
of solutions to a set of linear inequalities in a specified region of $\mathbb{R}^{m}$. In particular, we have shown that for the class of "regular" constant-rate hybrid systems Zenoness is equivalent to strong Zenoness; that is, the system has Zeno runs if if and only if all its runs are Zeno. In this case, it is clear that if Zeno runs exist, no safety controller exists.

When a system has Zeno runs but is not strongly Zeno, some legal controller exists. However we have shown that, contrary to earlier belief, the existence of a safety controller in Zeno systems does not always imply the existence of a minimally interventive (or minimally restrictive) controller. This implies, in particular, that the standard iterative synthesis algorithms that have been proposed in the literature may not apply in such cases. However, as was demonstrated, controllers can still be designed by more ad-hoc procedures.

We discussed some of the shortcomings of the approach for bypassing the problems associated with controller synthesis in Zeno systems that is based on introduction of switching delays. Specifically, the synthesis algorithm may not converge because of the introduced delay, and in cases where it converges, there may exist controllers less restrictive than the synthesized one.

We extended the study of Zenoness and existence of minimally interventive controllers to bounded-rate hybrid systems. Because of the nondeterminism in the dynamics of such systems, a gap appears between necessary conditions for Zenoness and sufficient conditions for Zenoness. Finally, we have shown that our analysis approach also applies to hybrid systems with nonlinear dynamics.

\section{References}

[1] R. Alur and D. Dill, 1990. Automata for modeling real-time systems. Proc. of the 17th International Colloquium on Automata, Languages and Programming, pp. 322-336.

[2] R. Alur, C. Courcoubetis, T. A. Henzinger, and P.-H. Ho, 1993. Hybrid automata: an algorithmic approach to the specification and verification of hybrid systems. Hybrid Systems, Lecture Notes in Computer Science, 736, Springer-Verlag, pp. 209-229.

[3] E. Azarin, O. Maler, and A. Pnueli, 1995. Symbolic controller synthesis for discrete and timed systems, Hybrid Systems II, Lecture Notes in Computer Science, 999, Springer Verlag, pp. 1-20.

[4] A. Balluchi, L. Benvenuti, T. Villa, H. Wong-Toi, A.L. Sangiovanni-Vincentelli, 1999. "Controller synthesis for hybrid systems with lower bounds of event separation", Proceedings 38th Conference on Decision and Control, Phoenix Az., pp. 3984-3989.

[5] A. Balluchi, L. Benvenuti, A.L. Sangiovanni-Vincentelli, T. Villa, H. Wong-Toi, 2000. "Non-Zeno controller synthesis for hybrid systems", preprint. 
[6] J.E.R. Cury, B. H. Krogh, and T. Niinomi, 1998. Synthesis of Supervisory Controllers for Hybrid Systems Based on Approximating Automata. IEEE Transactions on Automatic Control, 43(4), pp. 564-568.

[7] T. A. Henzinger, P. Kopke, A. Puri and P. Varaiya, 1995. What's decidable about hybrid automata, Proc. of the 27th Annual ACM Symposium on the Theory of Computing.

[8] T. A. Henzinger and H. Wong-Toi, 1996. Using HyTech to synthesize control parameters for a steam boiler. In Formal Methods for Industrial Applications: Specifying and Programming the Steam Boiler Control, Lecture Notes in Computer Science 1165, pp. 265-282, Springer-Verlag.

[9] T. A. Henzinger and P. W. Kopke, 1997. Discrete time control for rectangular hybrid automata. Proc. of 24 th International Colloquium on Automata, Languages and Programming.

[10] M. Heymann, F. Lin and G. Meyer, 1997. Control synthesis for a class of hybrid systems subject to configuration based safety constraints. NASA Technical Memorandum 112196.

[11] M. Heymann, F. Lin and G. Meyer, 1997. Synthesis of Minimally Restrictive Controllers for a Class of Hybrid Systems. in A. Nerode (Ed.), Hybrid Systems IV, Lecture Notes in Computer Science, Springer Verlag.

[12] M. Heymann, F. Lin and G. Meyer, 1998. Synthesis and viability of minimally interventive legal controllers for hybrid systems. Discrete Event Dynamic Systems: Theory and Applications, 8(2), pp. 105-135.

[13] M. Heymann F. Lin and G. Meyer, 2001. Control of Rate-Bounded Hybrid Systems with Liveness Specifications. in F. Colonius, U. Helmke, D. Pratzel-Wolters and F. Wirth, Eds., "Advances in Mathematical Systems Theory, pp. 151-168, Birkhauser publishers .

[14] K. H. Johansson, M. Egerstedt, J. Lygeros and S. Sastry, 1999. On the regularization of Zeno hybrid automata System \&6 Control Letters, 38, pp. 141-150.

[15] D. Kapur and R. K. Shyamasundar, 1997. Synthesizing Controllers for Hybrid Systems. Lecture Notes in Computer Science 1201, Springer Verlag, pp. 361-375.

[16] J. Lygeros, C. Tomlin and S. Sastry, 1999. Controllers for reachability specifications for hybrid systems. Automatica 35(3), pp. 349-370.

[17] J. Lygeros, K.H. Johansson, S. N. Simic, J. Zhang, and S. Sastry, 2003. Dynamical Properties of Hybrid Automata", IEEE. Trans. Automatic Control, Vol. 48, pp. 2-17. 
[18] O. Maler, Z. Manna and A. Pnueli, 1991. From timed to hybrid systems. In Real Time: Theory in Practice, Lecture Notes in Computer Science 600, pp. 447-484. Springer Verlag.

[19] O. Maler, A. Pnueli and J. Sifakis, 1995. On the synthesis of discrete controllers for timed systems. Lecture Notes in Computer Science 900, Springer-Verlag, pp. 229-242.

[20] A. S. Matveev and A. V. Savkin, 2000. Qualitative Theory of Hybrid Dynamical Systems, Birkuser Boston.

[21] A. Puri, V. Borkar and P. Varaiya, 1995. $\epsilon$-Approximation of Differential Inclusions, Proceedings of the 34th IEEE Conference on Decision and Control.

[22] A.V. Savkin and A.S. Matveev, 2000. Cyclic linear differential automata: A simple class of hybrid dynamical systems, Automatica, Vol. 36, pp. 727-734.

[23] A.V. Savkin and A.S. Matveev, 2000. Existence and Stability of Periodic Trajectories in Switched Server systems, Automatica, Vol. 36, pp. 775-779.

[24] S. Simic, K.H. Johansson, S. Sastry and J. Lygeros, Towards a Geometric Theory of Hybrid Systems. in Hybrid Systems: Computation and Control, B. Krogh and N. Lynch, Eds., pp. 421-436, LNCS 1790, Springer Verlag.

[25] H. Wong-Toi, 1997. The synthesis of controllers for linear hybrid automata. Proc. of 36th IEEE Conference on Decision and Control, pp. 4607-4613. Slobodan N. Simic, Karl Henrik Johansson,

[26] J. Zhang, K.H. Johansson, J. Lygeros and S. Sastry, 2001. Zeno Hybrid Systems. Intern. J. Robust and Nonlinear Control. Vol. 11 (5), pp. 435-451.

[27] J. Zhang, K.H. Johansson, J. Lygeros and S. Sastry, 2000. Dynamical Systems Revisited: Hybrid Systems with Zeno Executions, in Hybrid Systems: Computation and Control, B. Krogh and N. Lynch, Eds., pp. 451-464, LNCS 1790, Springer Verlag. 